\title{
NUMERICAL STUDY OF FISHER'S EQUATION BY A PETROV-GALERKIN FINITE ELEMENT METHOD
}

\author{
S. TANG' ${ }^{1}$ and R. O. WEBER ${ }^{2}$ \\ (Received 16 May 1990; revised 28 August 1990)
}

\begin{abstract}
Fisher's equation, which describes a balance between linear diffusion and nonlinear reaction or multiplication, is studied numerically by a Petrov-Galerkin finite element method. The results show that any local initial disturbance can propagate with a constant limiting speed when time becomes sufficiently large. Both the limiting wave fronts and the limiting speed are determined by the system itself and are independent of the initial values. Comparing with other studies, the numerical scheme used in this paper is satisfactory with regard to its accuracy and stability. It has the advantage of being much more concise.
\end{abstract}

\section{Introduction}

Fisher [4] introduced the equation

$$
u_{t}=\alpha u_{x x}+\beta u(1-u)
$$

to describe the propagation of a virile mutant in an infinitely long habitat. It also represents a model equation for the evolution of a neutron population in a nuclear reactor $[1,2]$ and a prototype model for a spreading flame $[7,13]$.

Equation (1) includes the effects of linear diffusion via $u_{x x}$ and nonlinear local multiplication or reaction via $u(1-u)$.

Re-scaling with the coordinates

$$
\tau=\beta t, \quad \xi=(\beta / \alpha)^{1 / 2} x,
$$

\footnotetext{
${ }^{1}$ Department of Mechanics, Peking University, Beijing 100871, China.

${ }^{2}$ Department of Mathematics, Australian Defence Force Academy, Canberra ACT 2600.

(C) Copyright Australian Mathematical Society 1991, Serial-fee code 0334-2700/91
} 
(1) becomes

$$
u_{\tau}=u_{\xi \xi}+u(1-u)
$$

We look for a steady-state travelling-wave solution of (2); that is

$$
u(\xi, \tau)=u(\xi-c \tau)
$$

A theoretical analysis (see, for example, [10]) predicts that $c \geq 2$. Furthermore, when $c=2$, the state will be stable, as Kolmogoroff et al [7] proved.

Gazdag and Canosa [5] have obtained numerical solutions of (2) using a pseudo-spectral approach and they show that if initial propagating waves with speed $c>2$ are specified, the speed will change into the minimum value $c=2$, and the final wave shape will be independent of the initial values.

Evans and Sahimi [3] used an AGE (alternating group explicit) iterative method to solve the reaction-diffusion equation

$$
u_{t}=\lambda u_{x x}+\mu u^{2}(1-u)
$$

and have obtained satisfactory results, of a qualitatively similar nature.

Both numerical schemes mentioned above are quite complicated and the pseudo-spectral approach always causes unexpected high-frequency oscillations, which must be filtered out at each time step.

Tang [11] introduced a Petrov-Galerkin FEM (finite element method) to solve dispersive wave equations, such as the Korteweg-deVries equation [11], the modified Korteweg-deVries equation [9], the regularised long wave equation [12], the rotation-modified Kadomtsev-Petviashvilli equation [6] and the forced Kadomtsev-Petviashvilli equation (Tang and Smyth, a private communication) with satisfactory accuracy, stability and conciseness.

Recently, we have expanded the use of this method to solve reactiondiffusion equations. Here, we describe the numerical scheme, using Fisher's equations as an example, and we present some interesting results.

\section{Numerical scheme}

The problem to be solved is

$$
\begin{aligned}
& u_{t}-\alpha u_{x x}-\beta u(1-u)=0, \quad-l<x<l, t>0, \\
& u(x, 0)=u_{0}(x) \\
& \lim _{|x| \rightarrow l} u(x, t)=0
\end{aligned}
$$


Define a piecewise linear function $\phi(x)$ and a cubic-spline function $\psi(x)$ by

$$
\begin{aligned}
& \phi(x)= \begin{cases}1-|x|, \quad|x| \leq 1, \\
0, \quad|x|>1,\end{cases} \\
& \psi(x)= \begin{cases}0, & x \leq-2, \\
(x+2)^{3} / 6, & -2<x \leq-1, \\
-\left(3 x^{3}+6 x^{2}-4\right) / 6, & -1<x \leq 0, \\
\left(3 x^{3}-6 x^{2}+4\right) / 6, & 0<x \leq 1, \\
-(x-2)^{3} / 6, & 1<x \leq 2, \\
0, & x>2 .\end{cases}
\end{aligned}
$$

The space $(-l, l)$ is then discretised, with the node points being

$$
x_{i}=-l+(i-1) h, \quad i=1, \ldots, N+1
$$

where $h=2 l / N$ is the spacing between node points.

As interpolating functions we use

$$
\phi_{i}(x)=\phi\left(\frac{x-x_{i}}{h}\right),
$$

and as weighting (test) functions we use

$$
\psi_{i}(x)=\psi\left(\frac{x-x_{i}}{h}\right)
$$

We now let

$$
U(x, t)=U_{j}(t) \phi_{j}(x) \quad j=1, \ldots, N+1
$$

and

$$
\tilde{U}^{2}(x, t)=U_{j}^{2}(t) \phi_{j}(x)
$$

stand for the terms linear in $u$ and the nonlinear $u^{2}$ term, respectively. The Einstein summation convention is assumed in these equations and in all following equations unless explicitly stated. Substituting into (4a) yields the error $\varepsilon(x, t)$ of our numerical approximation:

$$
\dot{U}_{j} \phi_{j}-\alpha U_{j} \phi_{j}^{\prime \prime}-\beta U_{j} \phi_{j}+\beta U_{j}^{2} \phi_{j}=\varepsilon(x, t)
$$

where ${ }^{\prime}$ and ' stand for derivatives with respect to $t$ and $x$, respectively.

We then take the inner product of (5) with the weighting function $\psi_{i}$, and let $\left(\varepsilon, \psi_{i}\right)=0$. This requires the error, due to discretisation, to vanish in the mean.

Considering ( $4 c$ ), and after integration by parts, we get

$$
M_{i j} \dot{U}_{j}-\left(Q_{i j}+\beta M_{i j}\right) U_{j}+\beta M_{i j} U_{j}^{2}=0, \quad i=1, \ldots, N+1
$$


where

$$
M_{i j}=\left\{\begin{array}{ll}
1, & j=i \\
13 / 33, & j=i+1 \\
1 / 66, & j=i \pm 2 \\
0, & \text { otherwise }
\end{array} \quad Q_{i j}=\frac{20 \alpha}{11 h^{2}} \begin{cases}-1, & j=i, \\
1 / 3, & j=i \pm 1 \\
1 / 6, & j=i \pm 2 \\
0, & \text { otherwise }\end{cases}\right.
$$

In (6) let

$$
L_{i j}=-\left(Q_{i j}+\beta M_{i j}\right), \quad N_{i j}=\beta M_{i j} .
$$

Then we can rewrite (6) as

$$
M_{i j} \dot{U}_{j}+L_{i j} U_{j}+N_{i j} U_{j}^{2}=0
$$

Using the trapezoidal rule to integrate this nonlinear ODE system yields

$$
M_{i j} U_{j}^{n+1}=M_{i j} U_{j}^{n}-\frac{\tau}{2}\left[L_{i j}\left(U_{j}^{n+1}+U_{j}^{n}\right)+N_{i j}\left\{\left(U_{j}^{n+1}\right)^{2}+\left(U_{j}^{n}\right)^{2}\right\}\right],
$$

where $\tau=$ time step, $U_{j}^{n}=U_{j}(n \tau)$.

Equation (8) is a nonlinear system of algebraic equations which is solved by the Newton-Raphson iteration procedure as follows. Begin by defining

$$
U_{j}^{n+1, k}=\text { value of } U_{j}^{n+1} \text { at } k \text { th iteration, }
$$

and

$$
N_{i j}\left(U_{j}^{n+1, k+1}\right)^{2}=N_{i j}\left(U_{j}^{n+1, k}\right)^{2}+2 N_{i j} U_{j}^{n+1, k}\left(U_{j}^{n+1, k+1}-U_{j}^{n+1, k}\right) .
$$

[Note: Hereafter $N_{i j} U_{j} V_{j}$ means $N_{i j} W_{j}$, where $W_{j}=U_{j} \times V_{j}$ and no summation convention is used in calculating $W_{j}$.] Substituting into (8) yields the linear system of algebraic equations

$$
\begin{aligned}
& \left\{M_{i j}+\frac{\tau}{2} L_{i j}+\tau N_{i j} U_{j}^{n+1, k}\right\} U_{j}^{n+1, k+1} \\
& =M_{i j} U_{j}^{n}-\frac{\tau}{2}\left[L_{i j} U_{j}^{n}+N_{i j}\left\{\left(U_{j}^{n}\right)^{2}-\left(U_{j}^{n+1, k}\right)^{2}\right\}\right],
\end{aligned}
$$

or in a simplified notation

$$
A_{i j} U_{j}^{n+1, k+1}=B_{i} \text {. }
$$

From the initial condition $u_{0}(x)$ we know $U_{j}^{0}=u_{0}\left(x_{j}\right)$, and we take $U_{j}^{1,1}=$ $U_{j}^{0}$, so we can get $U_{j}^{1,2}$, and so on, until

$$
\left|U_{j}^{1, k+1}-U_{j}^{1, k}\right|<\delta\left(=10^{-5} \text { in our program }\right) .
$$

This gives $U_{j}^{1}=U_{j}^{1, k+1}$ as output, which are then used as the initial values for calculating $U_{j}^{2}$, and so on. In this way we get $U_{j}^{n}$ step by step. Remember $U_{j}^{n}$ are just the numerical values of $u\left(x_{j}, n \tau\right)$. 
$A_{i j}$ is a banded and diagonally dominant matrix with bandwidth 5 , and hence (10) can be solved by Gaussian elimination.

Now we consider the consistency and accuracy of our numerical scheme. Let

$$
R(u)=(20 / 11)\left[u_{t}-\alpha u_{x x}-\beta u(1-u)\right],
$$

where $R$ is the differential operator in (4a) multiplied by a constant $20 / 11$, which is needed to make the main elements of $M_{i j}$ unity. Also let

$$
D(u)=M_{i j} \dot{U}_{j}+L_{i j} U_{j}+N_{i j} U_{j}^{2},
$$

in which $D$ is the operator for the discretisation of space in (7). Using the Taylor expansions

$$
\begin{aligned}
& f_{j \pm 1}=\sum_{n=0}^{5}( \pm 1)^{n} \frac{f_{j}^{(n)}}{n !} h^{n}+O\left(h^{6}\right), \\
& f_{j \pm 2}=\sum_{n=0}^{5}( \pm 1) \frac{f_{j}^{(n)}}{n !}(2 h)^{n}+O\left(h^{6}\right)
\end{aligned}
$$

for $\dot{U}_{j}, U_{j}$ and $U_{j}^{2}$ (which are the values of $U_{t}(x, t), U(x, t)$ and $U^{2}(x, t)$ at the nodal point $x_{j}$ at instant $t$ ), some straightforward calculations yield

$$
D(u)-R(u)=(5 / 11)[R(u)]_{x x j} h^{2}+O\left(h^{4}\right)
$$

where $[R(u)]_{x x j}$ are the second order partial derivatives of $R(u)$ with respect to $x$ calculated at $x_{j}$, and are, of course, equal to zero. Therefore, the truncation error in space discretisation is of the order $O\left(h^{4}\right)$. In other words, $D(u)$ is consistent with $R(u)$ when $h$ goes to zero and has fourth order accuracy.

On the other hand, it is well known that the trapezoidal rule in numerical integration in $t$ has truncation error $O\left(\tau^{2}\right)$. Putting them together, our numerical scheme is consistent with (4a) and has accuracy $O\left(\tau^{2}, h^{4}\right)$. If the Runge-Kutta method was used in integrating with respect to $t$, the accuracy could be raised to $O\left(\tau^{4}, h^{4}\right)$.

It can also be shown that the linearised version of our scheme in which $u^{2}$ is replaced by $u_{0} u$ with constant $u_{0} \in(0,1)$, is stable unconditionally, but it is of no use for our nonlinear problem, so the details are omitted. Various numerical experiments with $\tau$ and $h$ varying in a large range were performed to demonstrate the stability of our scheme.

\section{Numerical results}

For all the cases shown we set $\alpha=0.1, \beta=1.0, h=0.02, \tau=0.005$. The space scale $l$ is adjusted to ensure that there is sufficient space for waves to propagate. 
Two kinds of local initial values were used:

$$
u_{0}(x)=\operatorname{sech}^{2}(10 x) \text {, }
$$

with a sharp peak in the middle, and

$$
u_{0}(x)= \begin{cases}e^{10(x+1)}, & x \leq-1, \\ 1, & -1<x \leq 1, \\ e^{-10(x-1)}, & x>1,\end{cases}
$$

with a flat roof in the middle.

For both cases (A) and (B), the contour plots of $u$ at different $t$ are shown in Figures 1 to 6.

Figure 1 is for case (A) for a short period of time, showing the results from $t=0$ to $t=0.2$ with an increment $\Delta t=0.05$. At the very beginning, near $x=0, u_{x x}<0$ with a large absolute value, but the reaction term $u(1-u)$ is quite small, that is, the effect of diffusion dominates over the effect of reaction, so the peak goes down rapidly and gets flatter. A linearised analysis verifies this behaviour analytically.

Figure 2 is also for case (A), but the period of time is from $t=0$ to $t=5$ with $\Delta t=0.5$. It shows that after the peak of the contour arrives at the lowest level, the reaction term dominates the diffusion (gradually), so it begins to go up and flatten itself until, at the top, $u=1$.

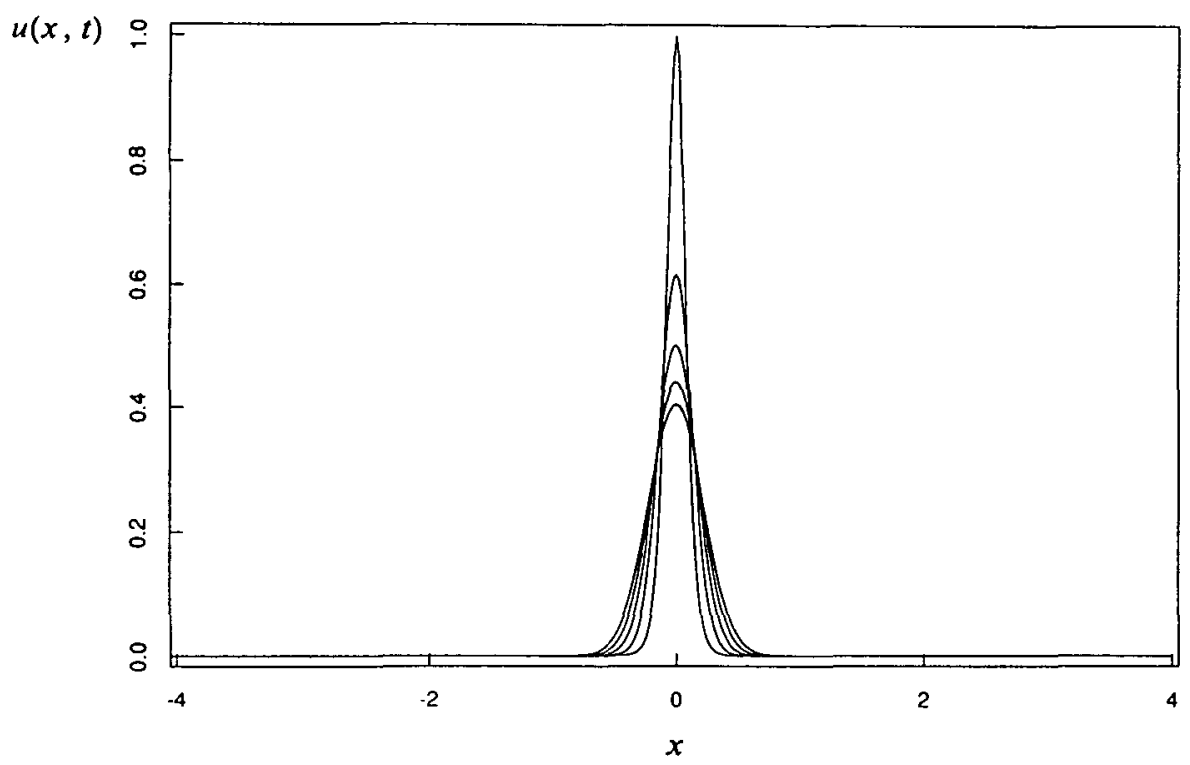

Figure 1. Contour plots of $u(x, t)$ for the case (A) from $t=0$ to $t=0.2$ with $\Delta t=0.05$. 


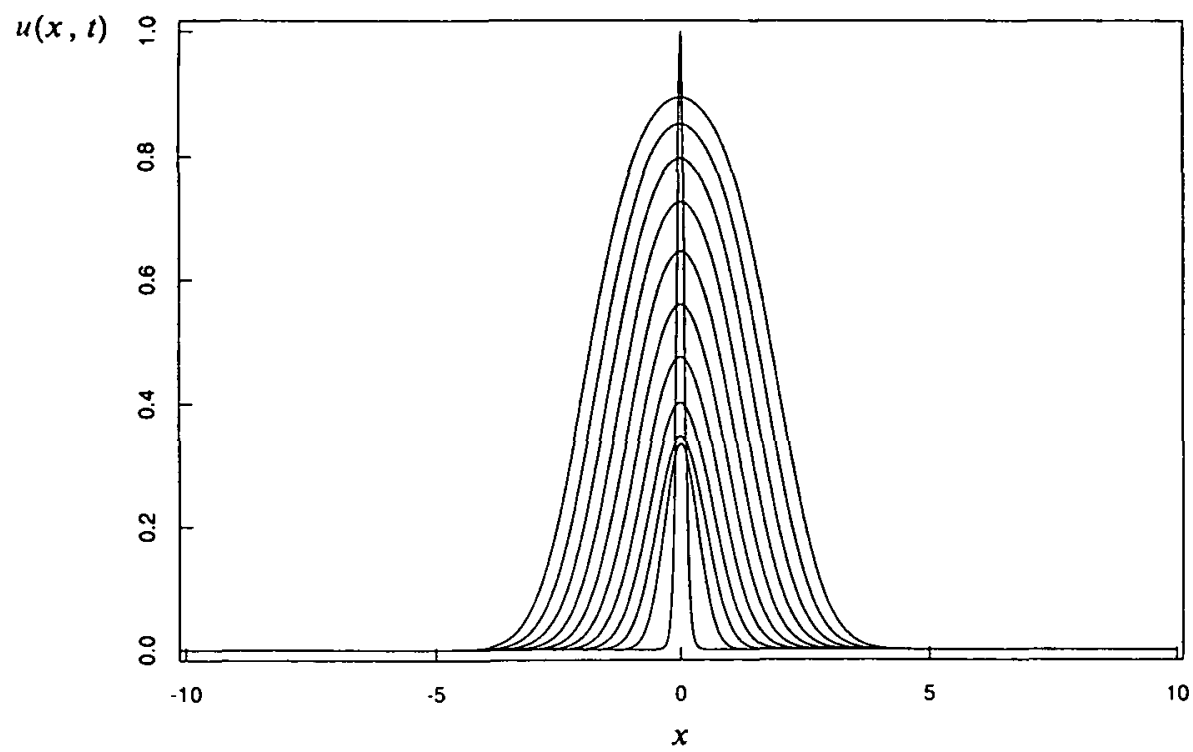

Figure 2. Contour plots of $u(x, t)$ for the case (A) from $t=0$ to $t=5$ with $\Delta t=0.5$.

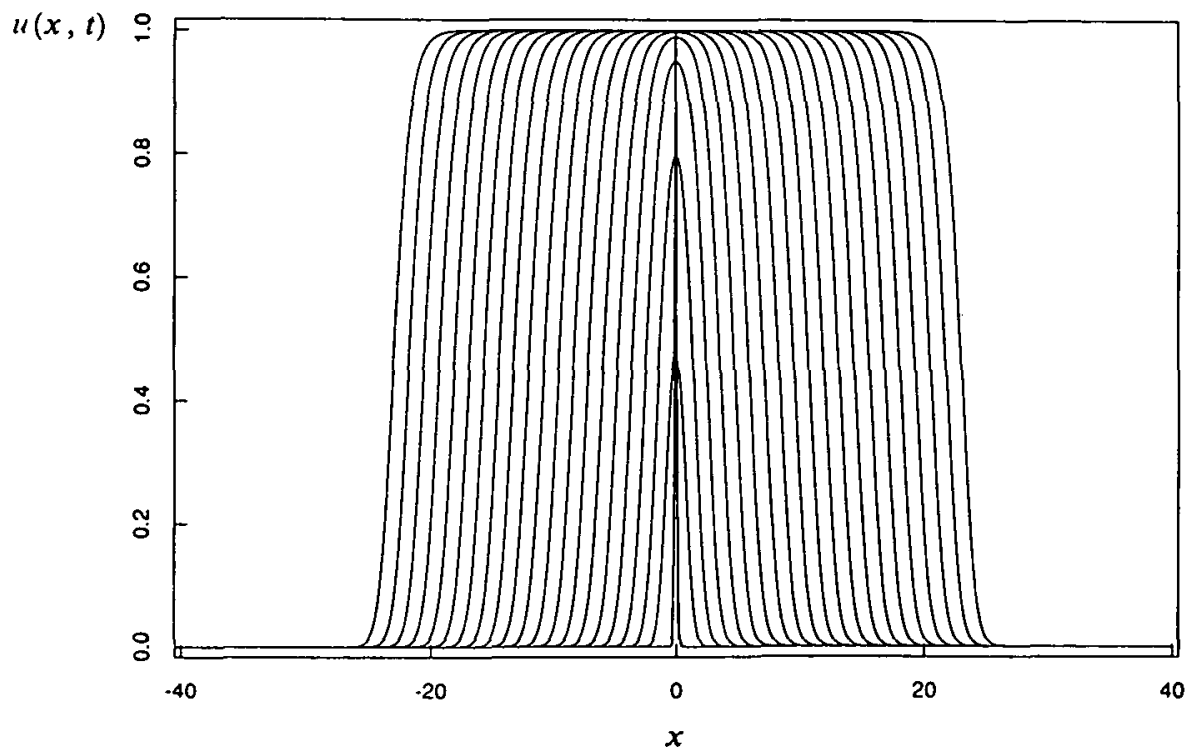

Figure 3. Contour plots of $u(x, t)$ for the case (A) from $t=0$ to $t=40$ with $\Delta t=2$.

Figure 3 is still for case (A), but it is for the period of time $t=0$ to $t=40$, with $\Delta t=2$. It shows that, after the peak has returned to its original position $u=1$, the contour on the top becomes flatter and flatter. So, after a long time, the contour looks like a bell with a flat top and very steep lateral 
sides, which propagate to the left and right symmetrically. The wave fronts (i.e. the lateral sides) approach a fixed shape and their propagating speed approaches a constant value $c$. Because the height of the contour is 1 , we can use half of the area under the contour to define the position of the wave fronts in the mean in the $x$-direction. The values of the speed calculated in this way at different $t$ keep on growing: at $t=36,38$ and 40, they are $0.61906,0.61983$ and 0.62053 ; and they are approaching the steady-state value of $2 \sqrt{\alpha \beta}=0.63246$, corresponding to our choice of $\alpha=0.1$ and $\beta=1.0$.

Figures 4, 5 and 6 show the stages of development of case (B). In contrast with case (A), Figure 4 shows, at the first stage, in the middle part, that both the effects of diffusion and reaction are very small, and the contours get rounded only at the edges, where the diffusion effects are larger than anywhere else. Affected by these, we can see in Figure 5 that the whole contour goes down a little bit, and then comes up. However, when time becomes large, Figure 6 shows that the limiting contour is almost the same as in case (A). This demonstrates that the limiting wave fronts and their propagating speed do not depend upon the initial values.

Finally, in Figure 7 we show the results for the initial condition

$$
u_{0}(x)=0.1 \operatorname{sech}^{2}(10 x)
$$

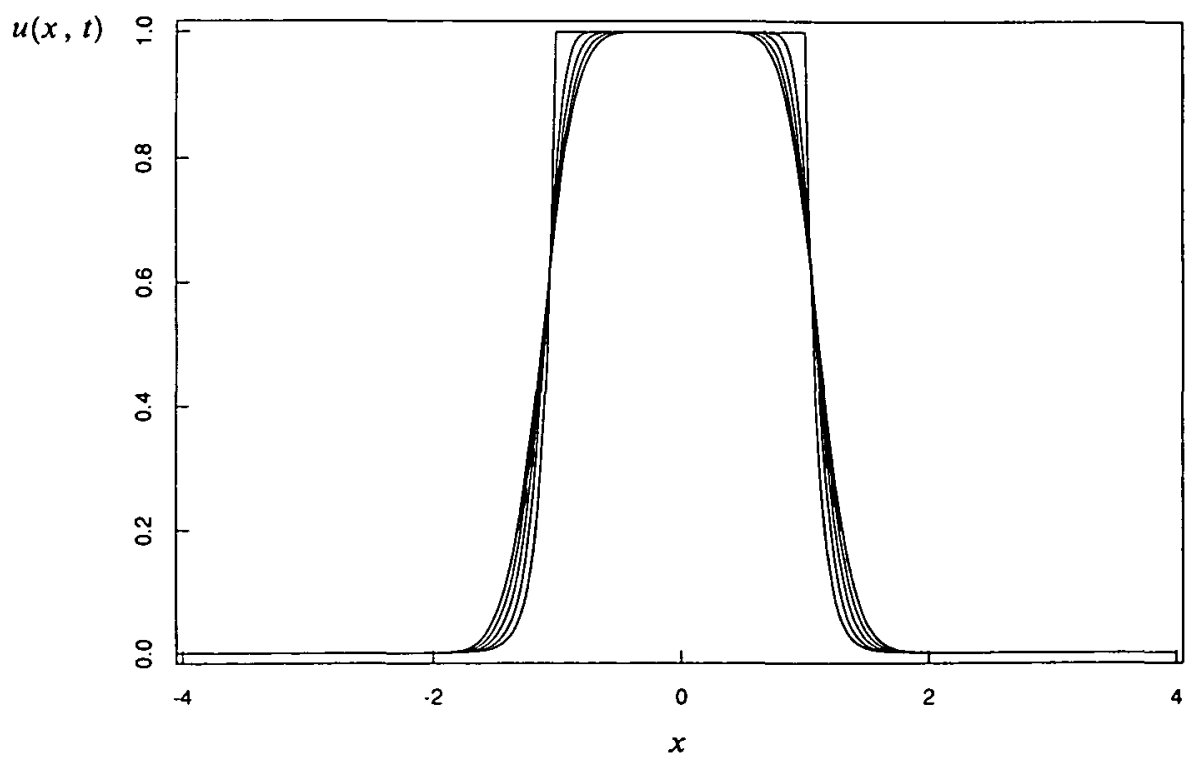

Figure 4. Contour plots of $u(x, t)$ for the case (B) from $t=0$ to $t=0.2$ with $\Delta t=0.05$. 


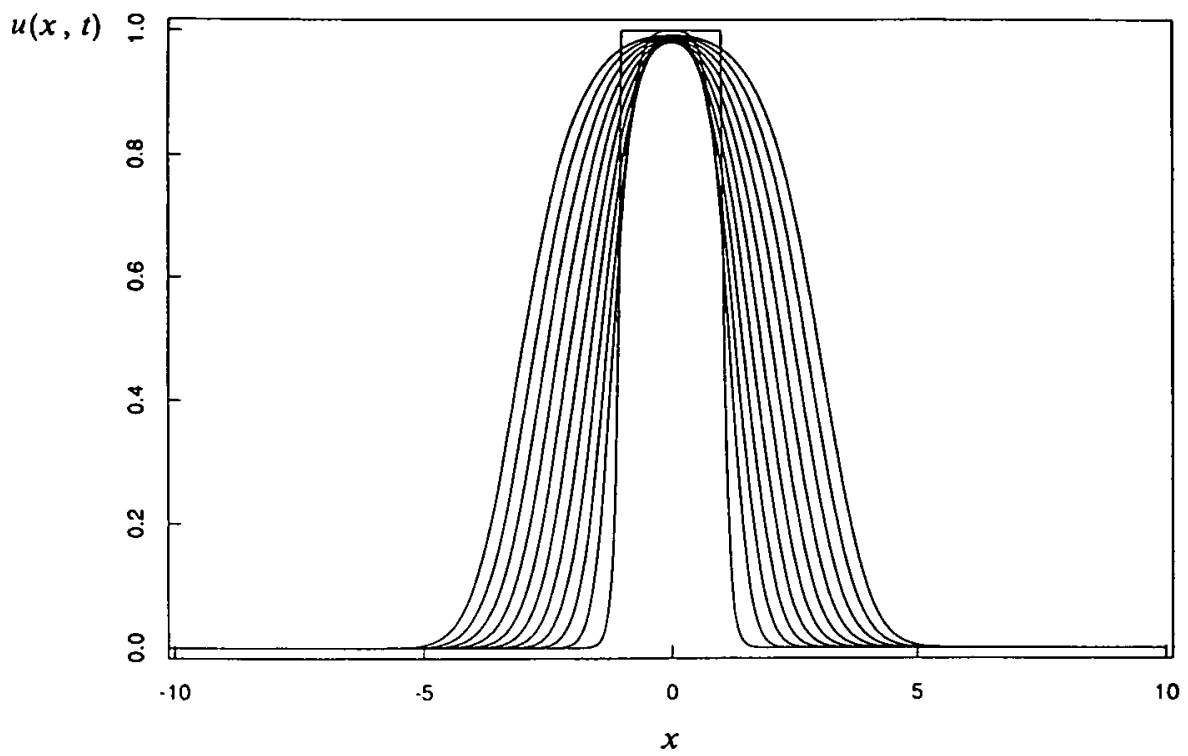

Figure 5. Contour plots of $u(x, t)$ for the case (B) from $t=0$ to $t=5$ with $\Delta t=0.5$.

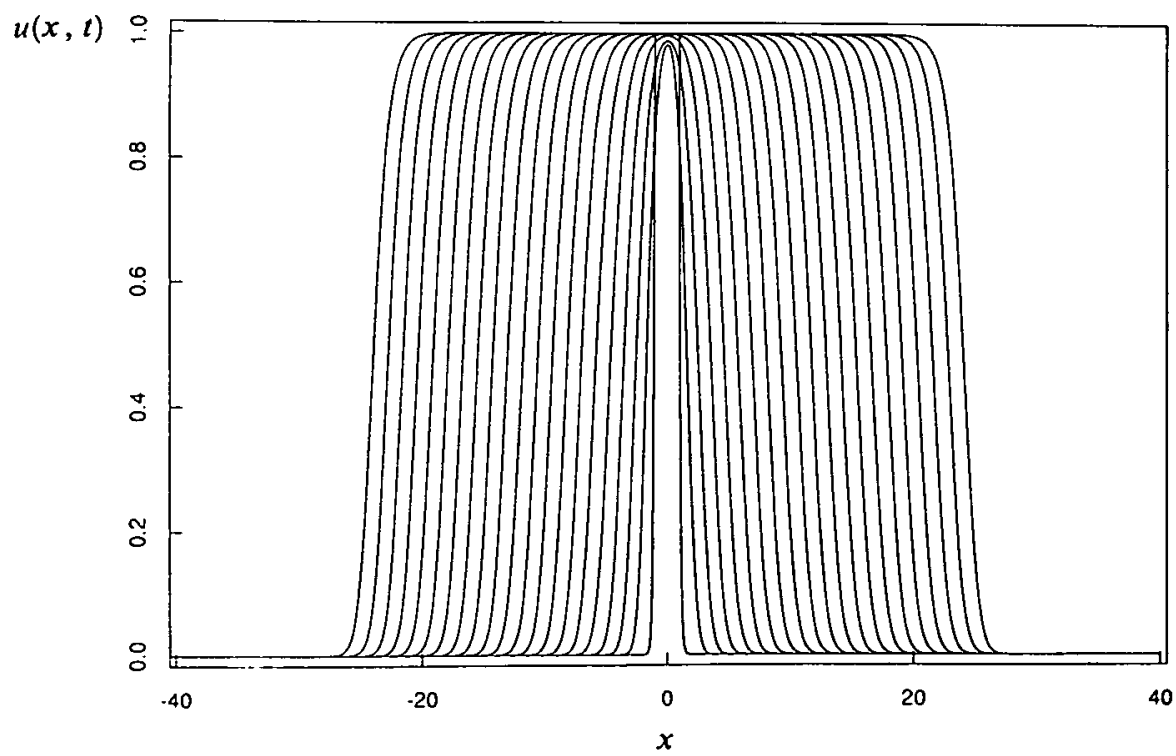

FIGURE 6. Contour plots of $u(x, t)$ for the case (B) from $t=0$ to $t=40$ with $\Delta t=2$.

which demonstrates how any small initial disturbance will also grow in time. This is because $u=0$ is an unstable equilibrium state of (1).

From all the figures shown, during the running of 8,000 time steps, we 


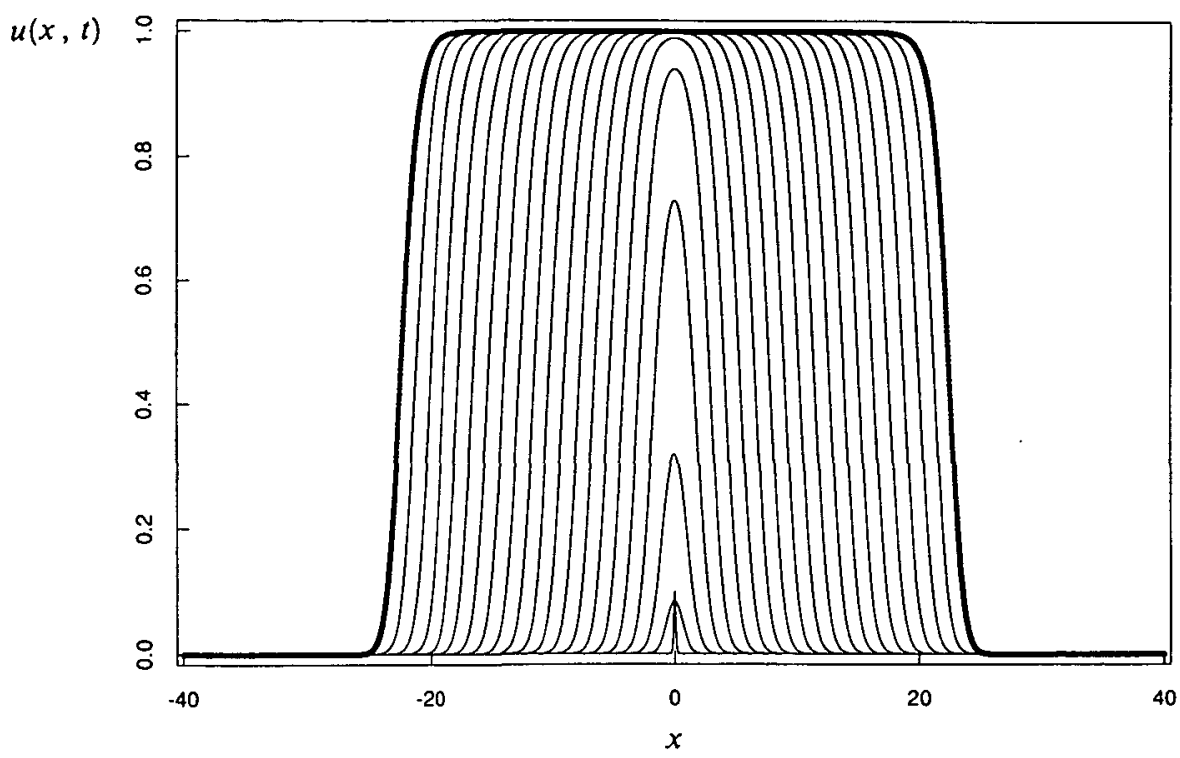

Figure 7. Contour plots of $u(x, t)$ for the case (C) from $t=0$ to $t=40$ with $\Delta t=2$.

could find no high frequency oscillatory tails caused by numerical errors, which is in contrast to what was reported by Smith [8] and Gazdag and Canosa [5] for their numerical methods. It shows that our numerical scheme is satisfactory in both accuracy and stability. Furthermore, comparing with both the pseudo-spectral and the AGE methods, the conciseness of our numerical scheme is quite obvious, as our Fortran program only takes two pages, including the standard subroutine of Gaussian elimination.

The reaction-diffusion equation (3) was also solved using our numerical scheme. In appearance, the contour plots for the reaction-diffusion in (3) are quite similar to those for Fisher's equation (1). But, if we use the initial condition (A), since the reaction near $x=0$ is less than that for Fisher's equation, the peak of $u$ goes down rapidly and comes up slowly. On the other hand, the wave fronts approach their limiting shape and propagating speed, (which equals 0.2236 when $\lambda=0.1$ and $\mu=1$ were chosen), much slower for the reaction-diffusion equations (3) than they did for Fisher's equation (1).

\section{Conclusions}

1. Fisher's equation, due to the balance of diffusion and multiplication, makes any local disturbance grow to the steady-state solution, $u=1$, which replace $u=0$ (a quasi-steady state) gradually. The interfaces between the two states (wavefronts) propagate with a constant limit- 
ing speed $c=2 \sqrt{\alpha \beta}$, which is determined by the system itself and is not affected by initial values.

2. In comparison with existing numerical schemes used to solve Fisher's equation, the scheme in this paper is an improvement in terms of stability and conciseness; and it has comparable accuracy.

3. This method, with some modifications, seems to be easily extended to solve model equations including more mechanical, physical or biophysical effects, such as nonlinear convection, reaction, linear diffusion and dispersion.

\section{Acknowledgement}

One of us (S. Tang) appreciates the support of the University College of the University of New South Wales, Australia, where this work was completed. The advice of Patrick Tang and Ian French on various computational issues is also appreciated.

\section{References}

[1] J. Canosa, "Diffusion in nonlinear multiplicative media", J. Math. Phys., 10 (1969) 1862-8.

[2] J. Canosa, "On a nonlinear diffusion equation describing population growth", IBM J. Res. Develop, 17 (1973) 307-13.

[3] D. J. Evans and M. S. Sahimi, "The alternating group explicit (AGE) iterative method to solve parabolic and hyperbolic partial differential equations", Ann. of Numerical Fluid Mechanics and Heat Transfer, 2 (1989) 283-389.

[4] R. A. Fisher, "The wave of advance of advantageous genes", Ann. of Eugen, 7 (1936) 355-69.

[5] J. Gazdag and J. Canosa, "Numerical solutions of Fisher's equation", J. Appl. Prob., 11 (1974) 445-57.

[6] R. Grimshaw and S. Tang, "The rotation-modified Kadomtsev-Petviashvili equation: an analytical and numerical study" (submitted) (1989).

[7] A. Kolmogoroff, I. Petrovsky and N. Piscounoff, "Study of the diffusion equation with growth of the quantity of matter and its application to biology problem", Bulletin de l'Université d'élat à Moscou, Série internationale, Section A, 1 (1937).

[8] G. D. Smith, (1978), Numerical solution of partial differential equations, (Oxford University Press, 1978).

[9] H. Sun and S. Tang, "Analytical and numerical studies on interaction of solitons of Modified KdV equations", Chinese Journal of Computational Physics, (in press).

[10] P. L. Sachdev, Nonlinear diffusive waves, (Cambridge University Press, pp. 103-5, 1987). 
[11] S. Tang, "Numerical study on KdV solitons and their interactions by a Petrov-Galerkin finite element method", Acta Mechanica, Sinica, 21 (1989) 354-9.

[12] S. Tang and W. Wang, "Numerical study on regularized long wave equations", Chinese Journal of Computational Physis, 3 (1989) 1989.

[13] J. B. Zeldovich and D. A. Frank-Kamenetzki, (1938), “A theory of thermal propagation of flame”, Acta Physicochimica U.R.S.S., 9 (1938) No. 2. 